Network Working Group

Request for Comments: 3340

Category: Standards Track
M. Rose

Dover Beach Consulting, Inc.

G. Klyne

Clearswift Corporation

D. Crocker

Brandenburg InternetWorking

July 2002

\title{
The Application Exchange Core
}

\section{Status of this Memo}

This document specifies an Internet standards track protocol for the Internet community, and requests discussion and suggestions for improvements. Please refer to the current edition of the "Internet Official Protocol Standards" (STD 1) for the standardization state and status of this protocol. Distribution of this memo is unlimited.

Copyright Notice

Copyright (C) The Internet Society (2002). All Rights Reserved.

Abstract

This memo describes Application Exchange (APEX) Core, an extensible, asynchronous message relaying service for application layer programs.

Table of Contents

1. Introduction . . . . . . . . . . . . . . . . . . . . 2

1.1 Overview . . . . . . . . . . . . . . . . . . . . . 3

1.2 Architecture at a Glance. . . . . . . . . . . . . . 4

2. Service Principles . . . . . . . . . . . . . . . . . 5

2.1 Modes of Operation . . . . . . . . . . . . . . . . . . 5

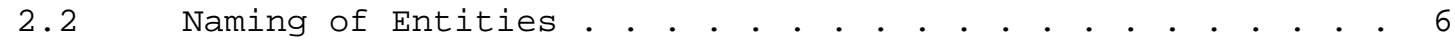

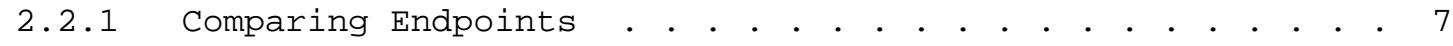

3. Service Provisioning . . . . . . . . . . . . . . . . . . 7

3.1 Connection Establishment . . . . . . . . . . . . . . 7

3.2 Authentication . . . . . . . . . . . . . . . . . 8

3.3 Authorization . . . . . . . . . . . . . . . . . 8

3.4 Confidentiality . . . . . . . . . . . . . . . . . . . . . 8

3.5 Relaying Integrity . . . . . . . . . . . . . . . 8

3.6 Traffic Analysis . . . . . . . . . . . . . . . . . 9

4. The APEX . . . . . . . . . . . . . . . . . . . . . . . . . 9

4.1 Use of XML and MIME . . . . . . . . . . . . . . . . . . . . 9

4.2 Profile Identification and Initialization . . . . . . . . 10

4.3 Message Syntax . . . . . . . . . . . . . . . . 11 


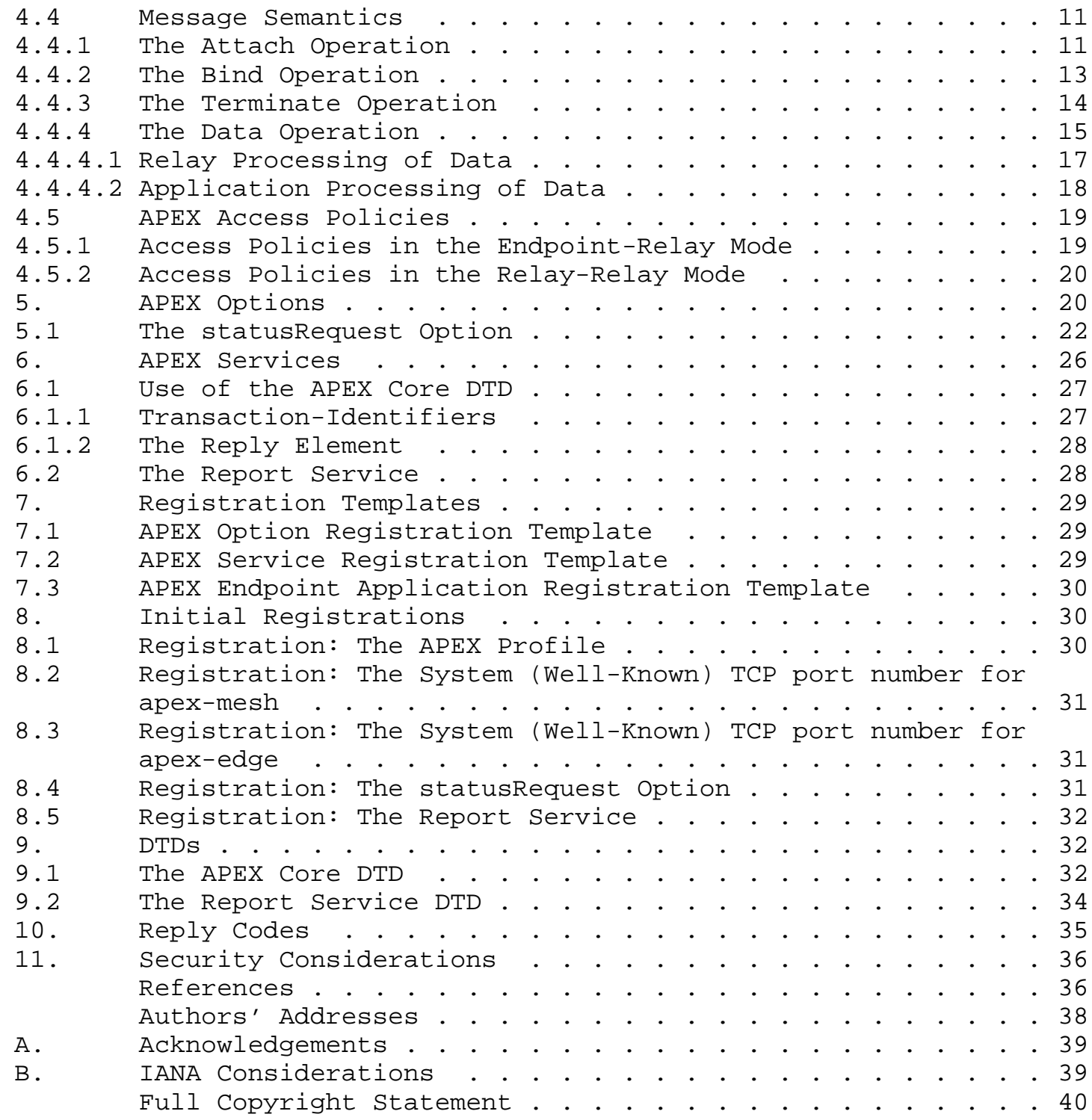

1. Introduction

Network applications can be broadly distinguished by five operational characteristics:

- server push or client pull;

o synchronous (interactive) or asynchronous (batch); 
o time-assured or time-insensitive;

o best-effort or reliable; and,

o stateful or stateless.

For example:

o the world-wide web is a pull, synchronous, time-insensitive, reliable, stateless service; whilst

- Internet mail is a push, asynchronous, time-insensitive, besteffort (without DSN), stateless service.

Messaging applications vary considerably in their operational requirements. For example, some messaging applications require assurance of timeliness and reliability, whilst others do not.

These features come at a cost, in terms of both infrastructural and configuration complexity. Accordingly, the underlying service must be extensible to support different requirements in a consistent manner.

This memo defines a core messaging service that supports a range of operational characteristics. The core service supports a variety of tailored services for both user-based and programmatic exchanges.

\subsection{Overview}

APEX provides an extensible, asynchronous message relaying service for application layer programs.

APEX, at its core, provides a best-effort datagram service. Each datagram, simply termed "data", is originated and received by APEX "endpoints" -- applications that dynamically attach to the APEX

"relaying mesh".

The data transmitted specifies:

o an originating endpoint;

- an opaque content (via a URI-reference);

o one or more recipient endpoints; and,

o zero or more options. 
Options are used to alter the semantics of the service, which may occur on a per-recipient or per-data basis, and may be processed by either a single or multiple relays.

Additional APEX services are provided on top of the relaying mesh; e.g., access control and presence information.

APEX is specified, in part, as a BEEP [1] "profile". Accordingly, many aspects of $\operatorname{APEX}$ (e.g., authentication) are provided within the BEEP core. Throughout this memo, the terms "peer", "initiator", "listener", "client", and "server" are used in the context of BEEP. In particular, section 2.1 of the BEEP core memo discusses the roles that a BEEP peer may perform.

When reading this memo, note that the terms "endpoint" and "relay" are specific to APEX, they do not exist in the context of BEEP.

1.2 Architecture at a Glance

The APEX stack:

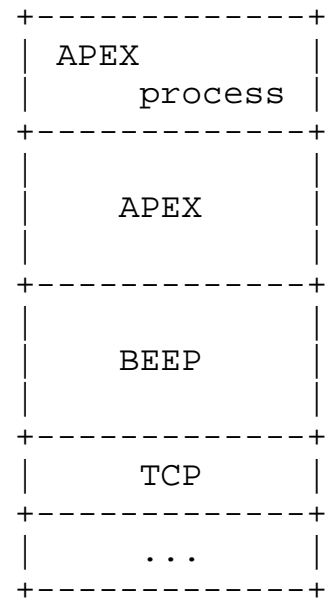

an APEX process is either:

- an application attached as an APEX endpoint; or,

- an APEX relay

APEX services are realized as applications having a special relationship with the APEX relays in their administrative domain 
The APEX entities:

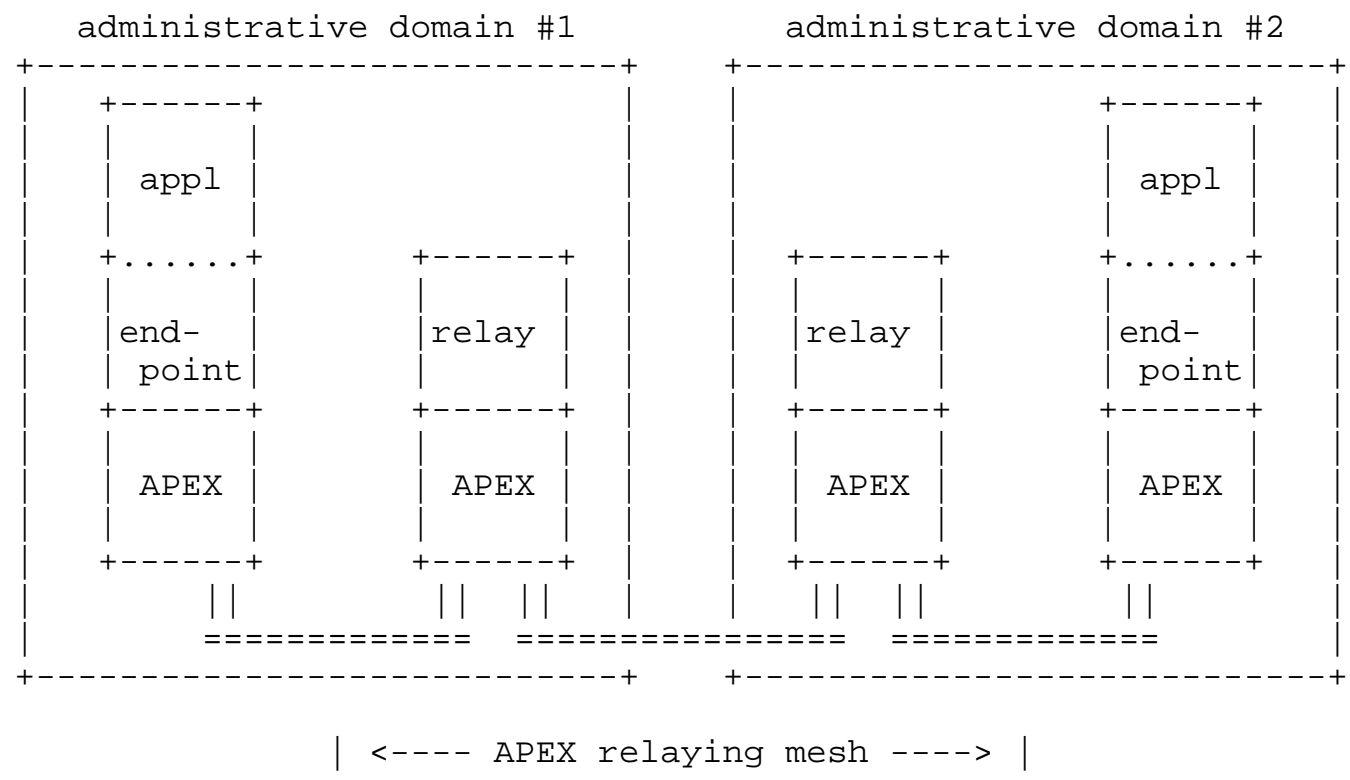

Note: relaying between administrative domains is configured using SRV RRs. Accordingly, the actual number of relays between two endpoints is not fixed.

2. Service Principles

\subsection{Modes of Operation}

APEX is used in two modes:

endpoint-relay: in which the endpoint is always the BEEP initiator of the service, whilst relays are always the BEEP listeners. In this context, applications attach as endpoints, and then the transmission of data occurs.

relay-relay: in which relays typically, though not necessarily, reside in different administrative domains. In this context, applications bind as relays, and then the transmission of data occurs.

In the endpoint-relay mode, an endpoint (BEEP initiator) may:

o attach as one or more endpoints;

o send data to other endpoints;

Rose, et. al.

Standards Track

[Page 5] 
o receive data from other endpoints; and,

o terminate any of its attachments.

A relay (BEEP listener), in addition to servicing requests from a BEEP initiator, may:

o terminate any of the endpoint's attachments;

o deliver data from other endpoints; and,

o indicate the delivery status of data sent earlier by the endpoint.

In the relay-relay mode, a relay (BEEP listener or initiator) may:

o bind as one or more administrative domains;

o send data;

o receive data; and,

o terminate any bindings.

2.2 Naming of Entities

Endpoints are named using the following ABNF [2] syntax:

; ; Domain is defined in [3], either a FQDN or a literal entity = local "@" Domain

local = address ["/" subaddress ]

address $\quad=$ token

subaddress $=$ token

; ; all non-control characters, excluding "/" and "@ " delimiters token $\quad=1 *(\% \times 20-2 \mathrm{E} / \% \times 30-3 \mathrm{~F} / \% \mathrm{x} 1-7 \mathrm{E} / \mathrm{UTF}-8) ;$; [ ]

Two further conventions are applied when using this syntax:

the "apex=" convention: All endpoint identities having a local-part starting with "apex=" are reserved for use by APEX services registered with the IANA; and, 
the "subaddress" convention: If the solidus character ("/", decimal code 47) occurs in the local-part, this identifies a subaddress of an endpoint identity (e.g., "fred/appl=wb@example.com" is a subaddress of the APEX endpoint "fred@example.com") .

All subaddresses starting with "appl=" are reserved for use by APEX endpoint applications registered with the IANA.

Relays, although not named, serve of behalf of administrative domains, as identified by a FQDN or a domain-literal, e.g., "example.com" or "[10.0.0.1]".

In APEX, "endpoints" and "relays" are the fundamental entities. APEX is carried over BEEP, which has the "peer" as its fundamental entity. The relationship between BEEP peer entities and APEX endpoint and relay entities are defined by APEX's Access Policies (Section 4.5).

\subsubsection{Comparing Endpoints}

Note that since the "local" part of an entity is a string of UTF-8 [4] octets, comparison operations on the "local" part use exact matching (i.e., are case-sensitive).

Accordingly, "fred@example.com" and "Fred@example.com" refer to different endpoints. Of course, relays serving the "example.com" administrative domain may choose to treat the two endpoints identically for the purposes of routing and delivery.

Finally, note that if an APEX endpoint is represented using a transmission encoding, then, prior to comparison, the encoding is reversed. For example, if the URL encoding is used, then "apex:fred@example.com" is identical to "apex:fo72ed@example.com".

3. Service Provisioning

3.1 Connection Establishment

The SRV algorithm [5] is used to determine the IP/TCP addressing information assigned to the relays for an administrative domain identified by a FQDN :

service: "apex-edge" (for the endpoint-relay mode), or "apex-mesh" (for the relay-relay mode);

protocol: "tcp"; and, domain: the administrative domain. 
If the administrative domain is identified by a domain-literal, then the IP address information is taken directly from the literal and the TCP port number used is assigned by the IANA for the registration in Section 8.2 .

\subsection{Authentication}

Authentication is a matter of provisioning for each BEEP peer (c.f., Section 4.5).

An APEX relay might be provisioned to allow a BEEP peer identity to coincide with a given endpoint identity. For example, a relay in the "example.com" administrative domain may be configured to allow a BEEP peer identified as "fred@example.com" to be authorized to attach as the APEX endpoint "fred@example.com".

\subsection{Authorization}

Authorization is a matter of provisioning for each BEEP peer (c.f., Section 4.5).

Typically, a relay requires that its BEEP peer authenticate as a prelude to authorization, but an endpoint usually does not require the same of its BEEP peer.

\section{4 Confidentiality}

Confidentiality is a matter of provisioning for each BEEP peer.

Typically, any data considered sensitive by an originating endpoint will have its content encrypted for the intended recipient endpoint(s), rather than relying on hop-by-hop encryption. Similarly, an originating endpoint will sign the content if end-toend authentication is desired.

\subsection{Relaying Integrity}

Data are relayed according to SRV entries in the DNS. Accordingly, relaying integrity is a function of the DNS and the applications making use of the DNS. Additional assurance is provided if the BEEP initiator requires that the BEEP listener authenticate itself. 


\subsection{Traffic Analysis}

Hop-by-hop protection of data transmitted through the relaying mesh (endpoint identities and content) is afforded at the BEEP level through the use of a transport security profile. Other traffic characteristics, e.g., volume and timing of transmissions, are not protected from third-party analysis.

4. The APEX

Section 8.1 contains the BEEP profile registration for APEX.

4.1 Use of XML and MIME

Each BEEP payload exchanged via APEX consists of an XML document and possibly an arbitrary MIME content.

If only an XML document is sent in the BEEP payload, then the mapping to a BEEP payload is straight-forward, e.g.,

C: $\operatorname{MSG} 12$. 11139

C: Content-Type: application/beep+xml

$\mathrm{C}:$

$C:$ <terminate transID $=^{\prime} 1^{\prime} />$

$C:$ END

Otherwise, if an arbitrary MIME content is present, it is indicated by a URI-reference [6] in the XML control document. The URIreference may contain an absolute-URI (and possibly a fragmentidentifier), or it may be a relative-URI consisting only of a fragment-identifier. Arbitrary MIME content is included in the BEEP payload by using a "multipart/related" [7], identified using a "cid" URL [8], and the XML control document occurs as the start of the "multipart/related", e.g.,

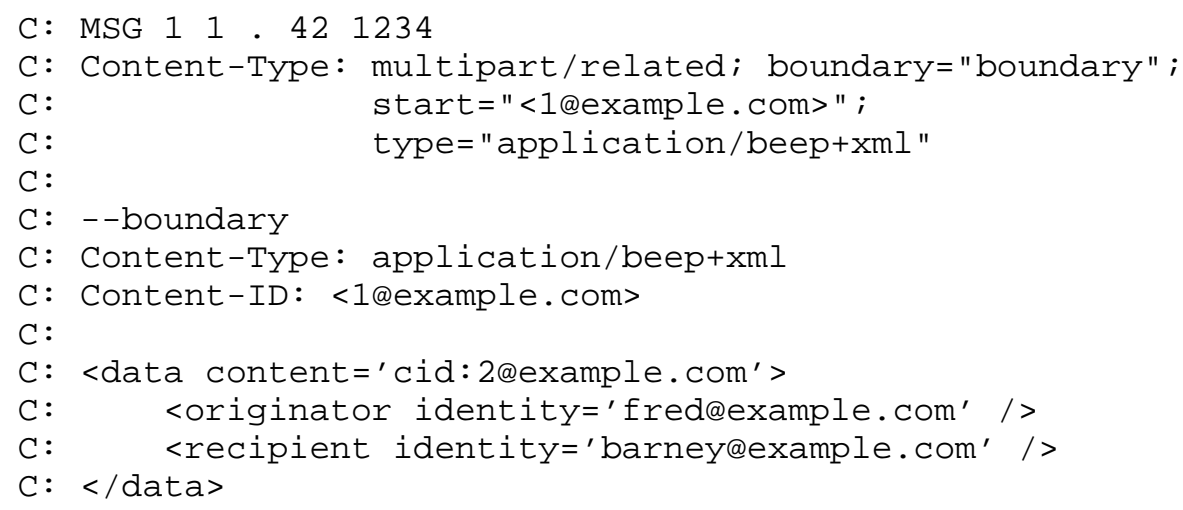




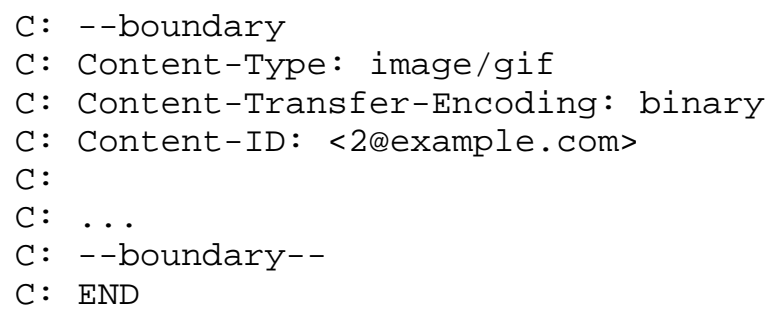

4.2 Profile Identification and Initialization

The APEX is identified as

http://iana.org/beep/APEX

in the BEEP "profile" element during channel creation.

No elements are required to be exchanged during channel creation; however, in the endpoint-relay mode, the BEEP initiator will typically include an "attach" element during channel creation, e.g., 


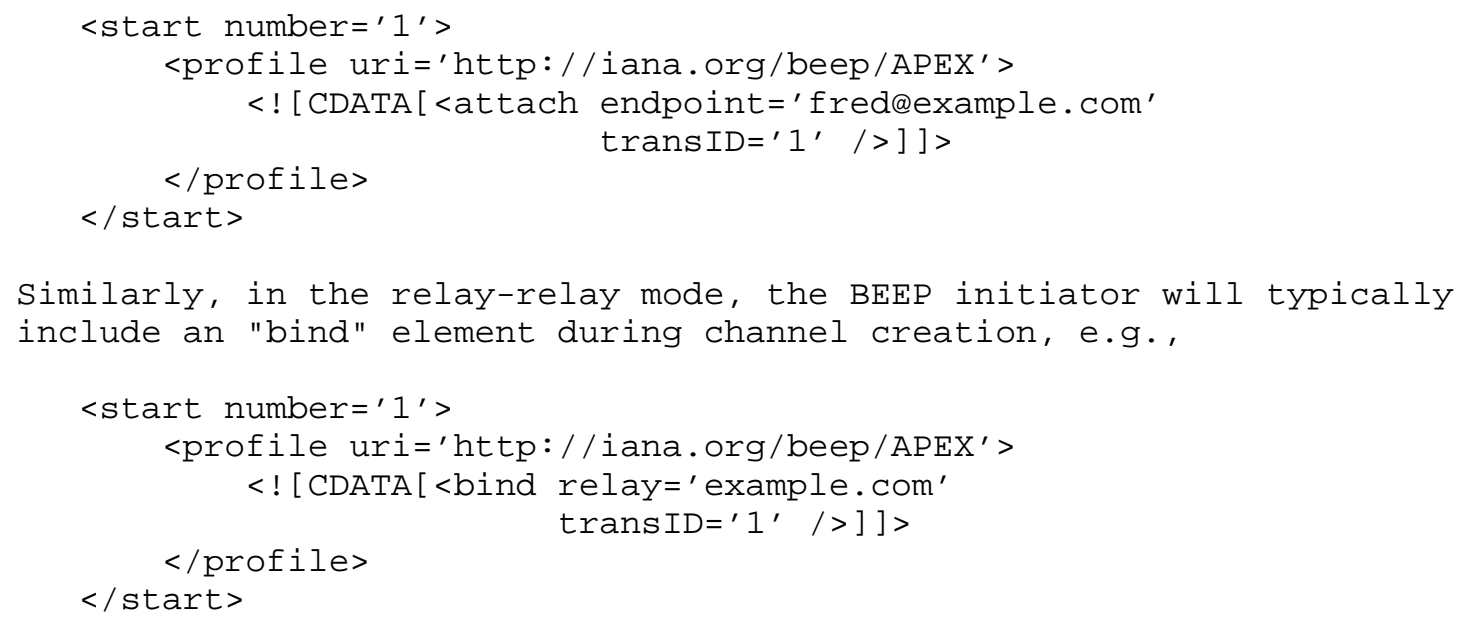

4.3 Message Syntax

Section 9.1 defines the BEEP payloads that are used in the APEX.

4. 4 Message Semantics

\subsubsection{The Attach Operation}

When an application wants to attach to the relaying mesh as a given endpoint, it sends an "attach" element to a relay, e.g.,

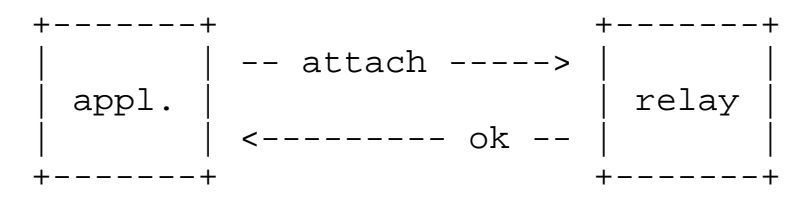

$\mathrm{C}:$ <attach endpoint=' fred@example. com' transID=' $1^{\prime} />$

S: $<$ ok />

or

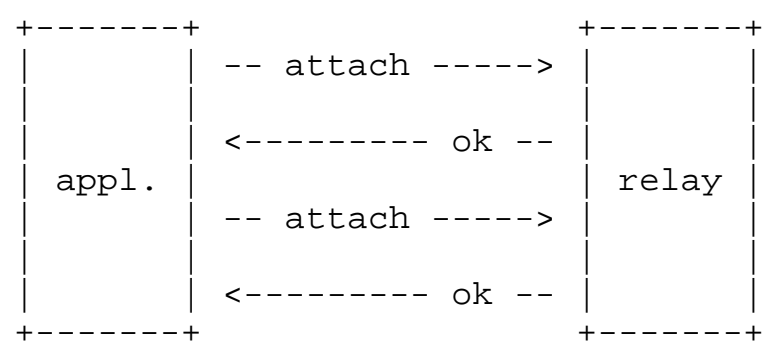

Rose, et. al.

Standards Track

[Page 11] 


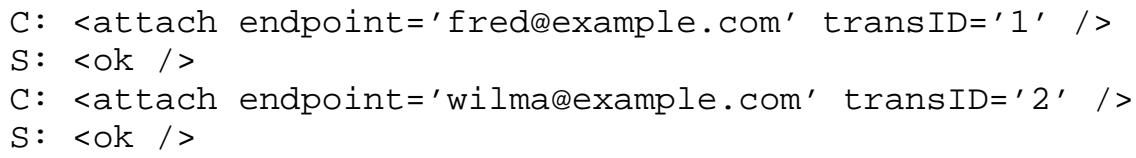

or

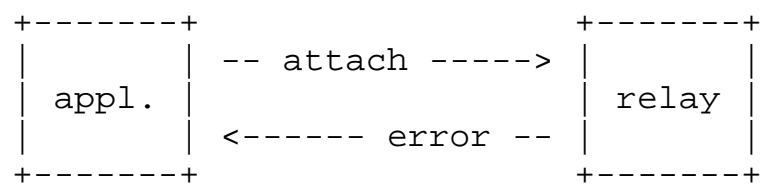

C: <attach endpoint=' fred@example.com' transID=' $1^{\prime} />$

$\mathrm{S}$ : <error code='537'>access denied</error>

The "attach" element has an "endpoint" attribute, a "transID"

attribute, and contains zero or more "option" elements:

- the "endpoint" attribute specifies the endpoint that the application wants to attach as;

o the "transID" attribute specifies the transaction-identifier associated with this operation; and,

o the "option" elements, if any, specify additional processing options (Section 5).

When a relay receives an "attach" element, it performs these steps:

1. If the transaction-identifier refers to a previous, non-terminated operation on this BEEP channel, an "error" element having code 555 is returned.

2. If the relay is in a different administrative domain than this endpoint, an "error" element having code 553 is returned.

3. If the application is not authorized to attach as this endpoint (c.f., Section 4.5.1), an "error" element having code 537 is returned.

4. If any options are present, they are processed.

5. If another application has already attached as this endpoint, an "error" element having code 554 is returned.

6. Otherwise, the application is bound as this endpoint, and an "ok" element is returned. 


\subsubsection{The Bind Operation}

When an application wants to identify itself as a relay, it sends a "bind" element to another relay, e.g.,

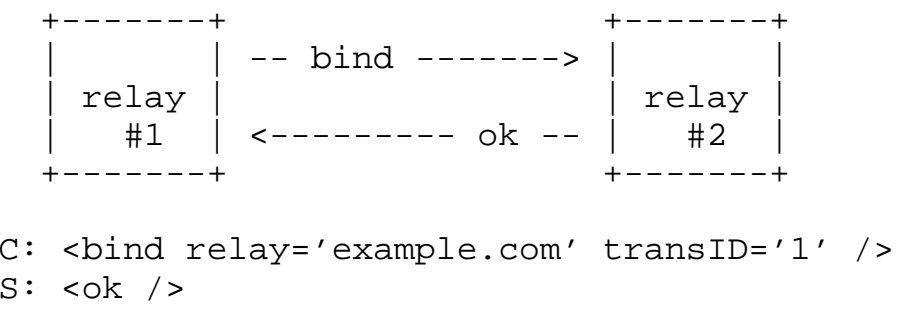

or
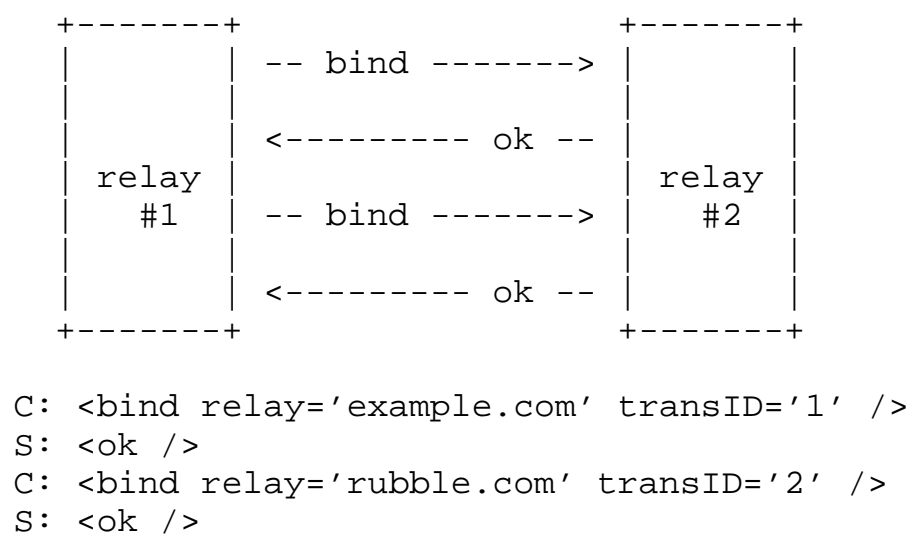

or

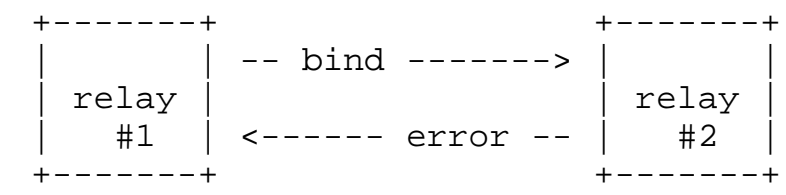

$\mathrm{C}:$ <bind relay=' example.com' transID=' $1^{\prime} />$

S: <error code='537'>access denied</error>

The "bind" element has a "relay" attribute, a "transID" attribute, and contains zero or more "option" elements:

- the "relay" attribute specifies the administrative domain on whose behalf the application wants to serve; 
- the "transID" attribute specifies the transaction-identifier associated with this operation; and,

o the "option" elements, if any, specify additional processing options (Section 5).

When a relay receives an "bind" element, it performs these steps:

1. If the transaction-identifier refers to a previous, non-terminated operation on this BEEP channel, an "error" element having code 555 is returned.

2. If the application is not authorized to bind on behalf of this administrative domain (c.f., Section 4.5.2), an "error" element having code 537 is returned.

3. If any options are present, they are processed.

4. Otherwise, the application is accepted as serving this administrative domain, and an "ok" element is returned.

\subsubsection{The Terminate Operation}

When an application or relay wants to release an attachment or binding, it sends a "terminate" element, e.g.,

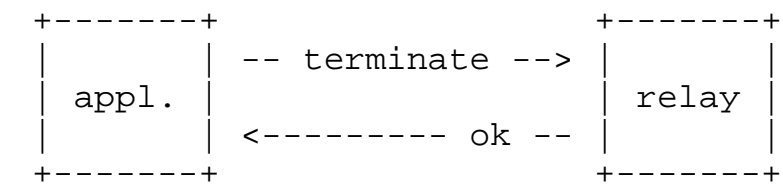

$C:$ <terminate transID $=^{\prime} 1^{\prime} />$

S: $<$ ok />

or

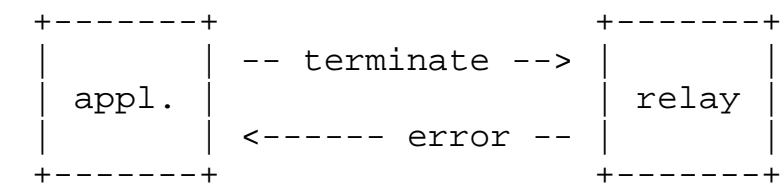

$\mathrm{C}:$ <terminate transID $=^{\prime} 13^{\prime} />$

$\mathrm{S}:$ <error code='550'>unknown transaction-identifier</error>

or 


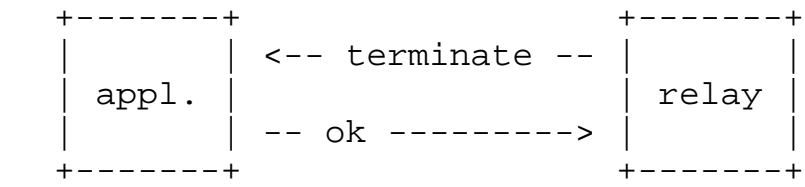

$C:$ <terminate transID=' $1^{\prime} />$

$\mathrm{S}:<\mathrm{ok} />$

The "terminate" element has a "transid" attribute, an optional "code" attribute, an optional "xml:lang" attribute, and may contain arbitrary textual content:

o the "transID" attribute specifies the transaction-identifier associated with this operation;

o the "code" attribute, if present, is a three-digit reply code meaningful to programs (c.f., Section 10);

o the "xml:lang" attribute, if present, specifies the language that the element's content is written in; and,

o the textual content is a diagnostic (possibly multiline) which is meaningful to implementers, perhaps administrators, and possibly even users.

When an application or relay receives a "terminate" element, it performs these steps:

1. If the value of the transaction-identifier is zero, then all associations established by this application over this BEEP session, either as an endpoint attachment or a relay binding, are terminated, and an "ok" element is returned.

2. Otherwise, if the transaction-identifier does not refer to a previous unterminated operation on this BEEP channel, an "error" element having code 550 is returned.

3. Otherwise, the application is no longer bound as an endpoint or a relay, and an "ok" element is returned.

\subsubsection{The Data Operation}

When an application or relay wants to transmit data over the relaying mesh, it sends a "data" element, e.g., 


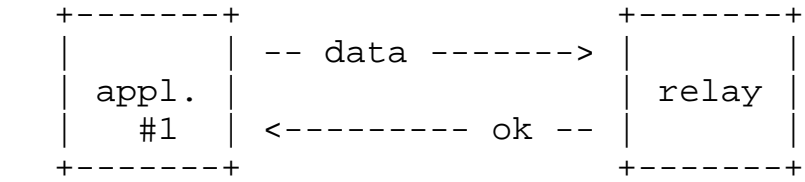

C: <data content=' cid:1@example.com'> <originator identity=' fred@example.com' /> $</$ data $>$

$\mathrm{S}:<\mathrm{ok} />$

or

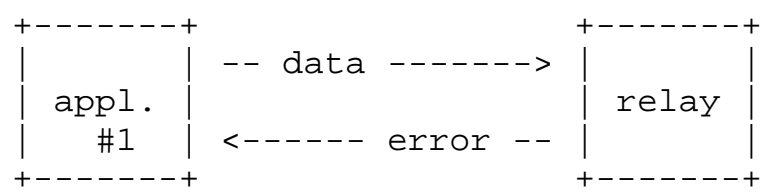

C: <data content=' cid:1@example.com'> <originator identity=' fred@example.com' /> <recipient identity='barney@example.com' /> $</$ data $>$

$S:$ <error code='537'>access denied</error>

or

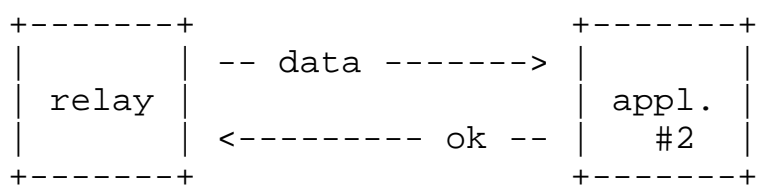

C: <data content=' cid:1@example.com'> <originator identity $=$ 'fredeexample.com' /> $</$ data $>$ <recipient identity='barney@example.com' />

$\mathrm{S}:<\mathrm{ok} />$

The "data" element has a "content" attribute, and contains an "originator" element, one or more "recipient" elements, zero or more "option" elements, and, optionally, a "data-content" element:

- the "content" attribute is a URI-reference that specifies the contents of the data (c.f., Section 4.1);

o the "originator" element refers to the endpoint sending the data; 
- each "recipient" element refers to an endpoint destination for the data;

o the "option" elements, if any, specify additional processing options (Section 5), termed per-data options; and,

o the "data-content" element, if present, specifies a nested XML entity that is referenced using a URI fragment-identifier as the value of the "content" attribute.

The "originator" element has an "identity" attribute, and contains zero or more option elements:

o the "identity" attribute specifies the sending endpoint; and

o the "option" elements, if any, specify additional processing options for the originator, termed per-originator options.

Each "recipient" element has an "identity" attribute, and contains zero or more option elements:

o the "identity" attribute specifies the destination endpoint; and

o the "option" elements, if any, specify additional processing options for this recipient, termed per-recipient options.

4.4.4.1 Relay Processing of Data

When a relay receives a "data" element, it performs these steps:

1. If the BEEP client is not authorized to originate or relay data on behalf of the "originator" endpoint (c.f., Section 4.5), an "error" element having code 537 is returned.

2. If any per-data options are present, they are processed.

3. An "ok" element is returned.

4. If any per-originator options are present, they are processed.

5. For each recipient:

1. If any per-recipient options are present, they are processed. 
2. If the recipient endpoint is not in the administrative domain associated with the relay, then an APEX session is established to a relay that accepts data for the recipient's administrative domain, and a new "data" element, containing that "recipient" element and all applicable options, is sent to that relay.

If an APEX session is established, the new "data" is sent, and the recipient's relay returns an "ok" element, then the recipient is considered to be successfully processed.

3. Otherwise, if the recipient endpoint is in the same administrative domain as the relay, the APEX access service must check that the originator endpoint is allowed to communicate with the recipient endpoint (the access entries [10] whose "owner" is the recipient must contain a "core:data" token for the originator), and the recipient endpoint must be currently attached.

If so, a new "data" element, containing only that "recipient" element, is sent to the corresponding application. If the recipient's endpoint returns an "ok" element, then the recipient is considered to be successfully processed.

Providing that these semantics are preserved, a relay may choose to optimize its behavior by grouping multiple recipients in a single "data" element that is subsequently transmitted.

Finally, note that a relay receiving a "data" element from an application may be configured to add administrative-specific options.

Regardless, all relays are expressly forbidden from modifying the content of the "data" element at any time.

\subsubsection{Application Processing of Data}

When an application receives a "data" element, it performs these steps:

1. If any per-data or per-originator options are present, they are not processed (but may be noted).

2. For each recipient:

1. If any per-recipient options are present, they are not processed (but may be noted).

2. If the application is not attached as the recipient endpoint, then an error in processing has occurred. 
3. Otherwise, the "data" element is further processed in an application-specific manner, and the recipient is considered to be successfully processed.

3. If no recipients could be successfully processed, an "error" element is returned; otherwise, an "ok" element is returned.

\subsection{APEX Access Policies}

Access to APEX is provided by the juxtaposition of:

- authenticating as a BEEP peer;

- attaching as an APEX endpoint or binding as an APEX relay; and,

o being listed as an actor by the APEX access service (c.f., [10]).

Each of these activities occurs according to the policies of the relevant administrative domain:

- each administrative domain is responsible for keeping its own house in order through "local provisioning"; and,

- each administrative domain decides the level of trust to associate with other administrative domains.

4.5.1 Access Policies in the Endpoint-Relay Mode

- When an application wants to attach to the relaying mesh, local provisioning maps BEEP peer identities to allowed APEX endpoints (c.f., Step 3 of Section 4.4.1).

Typically, the identity function is used, e.g., if an application authenticates itself as the BEEP peer named as "fred@example.com", it is allowed to attach as the APEX endpoint named as

"fred@example.com".

However, using the "subaddress" convention of Section 2.2, an application authorized to attach as a given APEX endpoint is also authorized to attach as any subaddress of that APEX endpoint, e.g., an application authorized to attach as the APEX endpoint "fred@example.com" is also authorized to attach as the APEX endpoint "fred/appl=wb@example.com" .

- When an application wants to send data, local provisioning maps attached endpoints to allowed originators (c.f., step 1 of section 4.4.4.1). 
Typically, the identity function is used, e.g., if an application attaches as the APEX endpoint named as "fred@example.com", it is allowed to send data originating from the same APEX endpoint. However, other policies are permissible, for example, the administrative domain may allow the application attached as the APEX endpoint named as "wilma@example.com" to send data originating as either "wilma@example.com" or "fred@example.com".

- Finally, when a relay is delivering to an endpoint within its own administrative domain, it consults the recipient's access entry looking for an entry having the originator as an actor (c.f., step 5.3 of Section 4.4.4.1).

\subsubsection{Access Policies in the Relay-Relay Mode}

- When an application wants to bind as a relay on behalf of an administrative domain, local provisioning may map BEEP peer identities to allowed APEX relays (c.f., Step 3).

If so, then typically the identity function is used. e.g., if an application authenticates itself as the BEEP peer named as "example.com", it is allowed to bind as a relay on behalf of the administrative domain "example.com".

- When a relay is sending data, no access policies, per se, are applied.

- When a relay is receiving data, local provisioning maps BEEP peer identities to allowed originators (c.f., step 1 of section 4.4 .4 .1 ).

Typically, the identity function is used, e.g., if a relay authenticates itself as being from the same administrative domain as the originator of the data, then the data is accepted.

In addition, some relays may also be configured as "trusted" intermediaries, so that if a BEEP peer authenticates itself as being from such a relay, then the data is accepted.

5. APEX Options

APEX, at its core, provides a best-effort datagram service. Options are used to alter the semantics of the core service.

The semantics of the APEX "option" element are context-specific. Accordingly, the specification of an APEX option must define:

o the identity of the option; 
o the context in which the option may appear;

o what content, if any, is contained within the option; and,

o the processing rules for the option.

An option registration template (Section 7.1) organizes this information.

An "option" element is contained within either a "data", "originator", "recipient", or an "attach" element, all of which are termed the "containing" element. The "option" element has several attributes and contains arbitrary content:

o the "internal" and the "external" attributes, exactly one of which is present, uniquely identify the option;

o the "targetHop" attribute specifies which relays should process the option;

o the "mustunderstand" attribute specifies whether the option, if unrecognized, must cause an error in processing to occur;

o the "transID" attribute specifies a transaction-identifier for the option; and,

o the "localize" attribute, if present, specifies one or more language tokens, each identifying a desirable language tag to be used if textual diagnostics are returned to the originator.

Note that if the containing element is an "attach", then the values of the "targetHop" and "transID" attributes are ignored.

The value of the "internal" attribute is the IANA-registered name for the option. If the "internal" attribute is not present, then the value of the "external" attribute is a URI or URI with a fragmentidentifier. Note that a relative-URI value is not allowed.

The "targetHop" attribute specifies which relay(s) should process the option:

this: the option applies to this relay, and must be removed prior to transmitting the containing element.

final: the option applies to this relay, only if the relay will transmit the containing element directly to the recipient. 
all: the option applies to this relay and is retained for the next.

Note that a final relay does not remove any options as it transmits the containing element directly to the recipient.

The "mustunderstand" attribute specifies whether the relay may ignore the option if it is unrecognized, and is consulted only if the "targethop" attribute indicates that the option applies to that relay. If the option applies, and if the value of the "mustUnderstand" attribute is "true", and if the relay does not "understand" the option, then an error in processing has occurred.

5.1 The statusRequest Option

Section 8.4 contains the APEX option registration for the "statusRequest" option.

If this option is present, then each applicable relay sends a "statusResponse" message to the originator. This is done by issuing a data operation whose originator is the report service associated with the issuing relay, whose recipient is the endpoint address of the "statusRequest" originator, and whose content is a

"statusResponse" element.

A "statusRequest" option MUST NOT be present in any data operation containing a "statusResponse" element. In general, applications should be careful to avoid potential looping behaviors if an option is received in error.

Consider these examples:

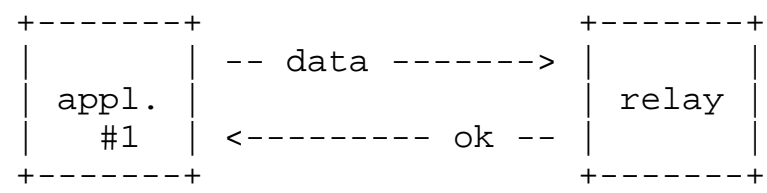

C: <data content=' cid:1 dexample.com'> <originator identity=' fredeexample.com' /> <recipient identity='barneydexample.com' /> <option internal=' statusRequest' targetHop='final' mustunderstand $=^{\prime}$ true' transID $=^{\prime} 86^{\prime} />$ $</$ data $>$

S : $<$ ok $/>$ 


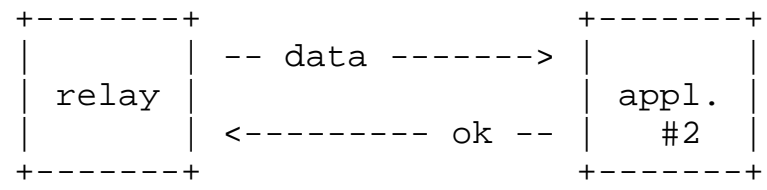

C: <data content=' cid:1 dexample.com'> <originator identity $=$ ' fredeexample.com' /> <recipient identity='barney@example.com' /> <option internal=' statusRequest' targetHop=' final' $</$ data $>$ mustUnderstand $=^{\prime}$ true' transID $=^{\prime} 86^{\prime} />$

$\mathrm{S}:<\mathrm{ok} />$

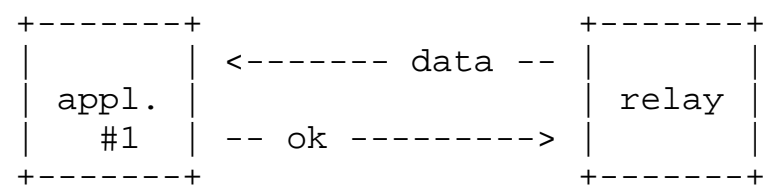

C: <data content=' \#Content'> <originator identity='apex=reporteexample.com' /> <recipient identity=' fred@example.com' /> $<$ data-content Name='Content'> <statusResponse transID $=^{\prime} 86^{\prime}>$ <destination identity='barney@example.com'> <reply code='250' /> $</$ destination $>$ $</$ statusResponse $>$ $</$ data-content $>$

$</$ data $>$

$\mathrm{S}:<\mathrm{ok} />$

or

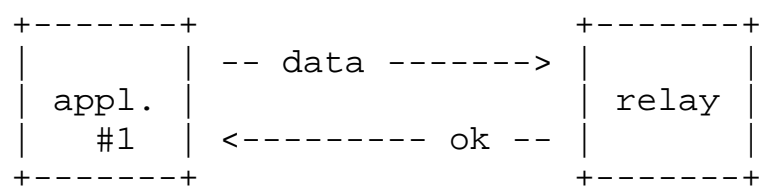

C: <data content='cid:1@example.com'> <originator identity=' fredeexample.com' /> <recipient identity='barney@example.com' /> <option internal=' statusRequest' targetHop=' final' mustUnderstand=' true' transID $={ }^{\prime} 86^{\prime} />$

$</$ data $>$

$\mathrm{S}:<\mathrm{ok} />$

Rose, et. al.

Standards Track

[Page 23] 


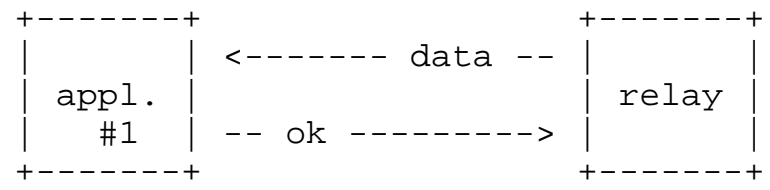

C: <data content=' \#Content'> <originator identity=' apex=report@example.com' /> <recipient identity=' fred@example.com' /> <data-content Name=' Content'> <statusResponse transID $=^{\prime} 86^{\prime}>$ <destination identity='barney@example.com'> <reply code='550'>unknown endpoint $</$ destination $>$ identity</reply $>$ $</$ statusResponse $>$

$</$ data $>$

$\mathrm{S}:<\mathrm{ok} />$

or

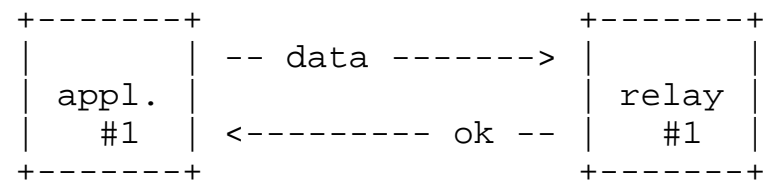

C: <data content=' cid:1@example.com'> <originator identity=' fred@example.com' /> <recipient identity='barneyarubble.com' />

coption internal=' statusRequest' targetHop='final' $</$ data $>$ mustUnderstand=' true' transID=' 86' />

$\mathrm{S}:<\mathrm{ok} />$

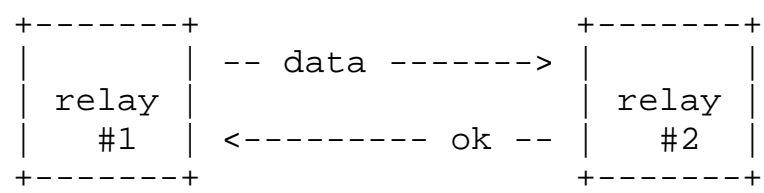

C: <data content=' cid:1@example.com'> <originator identity=' fred@example.com' /> <recipient identity='barney@rubble.com' /> <option internal=' statusRequest' targetHop=' final' $</$ data $>$ mustUnderstand=' true' transID $={ }^{\prime} 86^{\prime} />$

$\mathrm{S}:<\mathrm{ok} />$ 


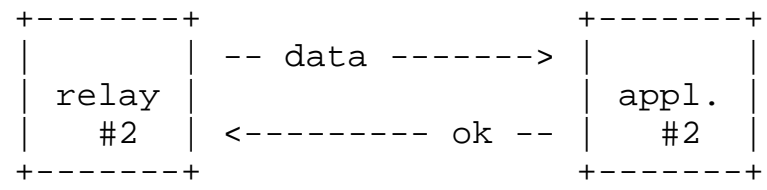

C: <data content=' cid:1 dexample.com'> <originator identity $=$ ' fred@example.com' /> <recipient identity='barneydexample.com' /> coption internal=' statusRequest' targetHop='final' $</$ data $>$ mustUnderstand $=^{\prime}$ true' transID $=^{\prime} 86^{\prime} />$

$\mathrm{S}:<\mathrm{ok} />$

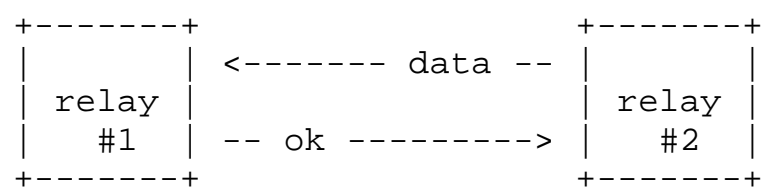

C: <data content=' \#Content'>

<originator identity=' apex=reporterubble.com' />

<recipient identity=' fred@example.com' />

$<$ data-content Name='Content'>

<statusResponse transID $=^{\prime} 86^{\prime}>$

<destination identity $=^{\prime}$ barneydrubble.com'> <reply code='250' /> $</$ destination $>$

$</$ statusResponse $>$

$</$ data-content $>$

$</$ data $>$

$\mathrm{S}:<\mathrm{ok} />$

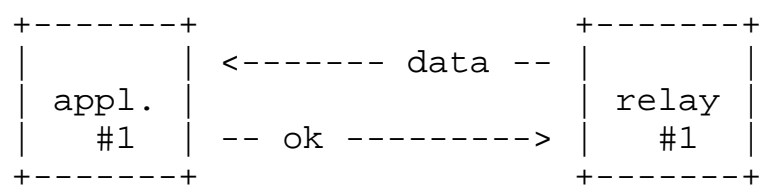

C: <data content=' \#Content'>

<originator identity=' apex=reportarubble.com' />

<recipient identity=' fred@example.com' />

<data-content Name='Content'>

<statusResponse transID $=^{\prime}$ 86'>

<destination identity $=^{\prime}$ barney@rubble.com' > <reply code='250' />

$</$ destination $>$

$</$ statusResponse $>$

Rose, et. al.

Standards Track

[Page 25] 


$$
\begin{aligned}
& \text { </data-content> } \\
& \text { </data> } \\
& \mathrm{S}:<\text { ok /> }
\end{aligned}
$$

Note that a trace of a data's passage through the relaying mesh can be achieved by setting the "targetHop" attribute to "all".

6. APEX Services

APEX, at its core, provides a best-effort datagram service. Within an administrative domain, all relays must be able to handle messages for any endpoint within that administrative domain. APEX services are logically defined as endpoints but, given their ubiquitous semantics, they do not necessarily need to be associated with a single physical endpoint. As such, they may be provisioned coresident with each relay within an administrative domain, even though they are logically provided on top of the relaying mesh, i.e.,

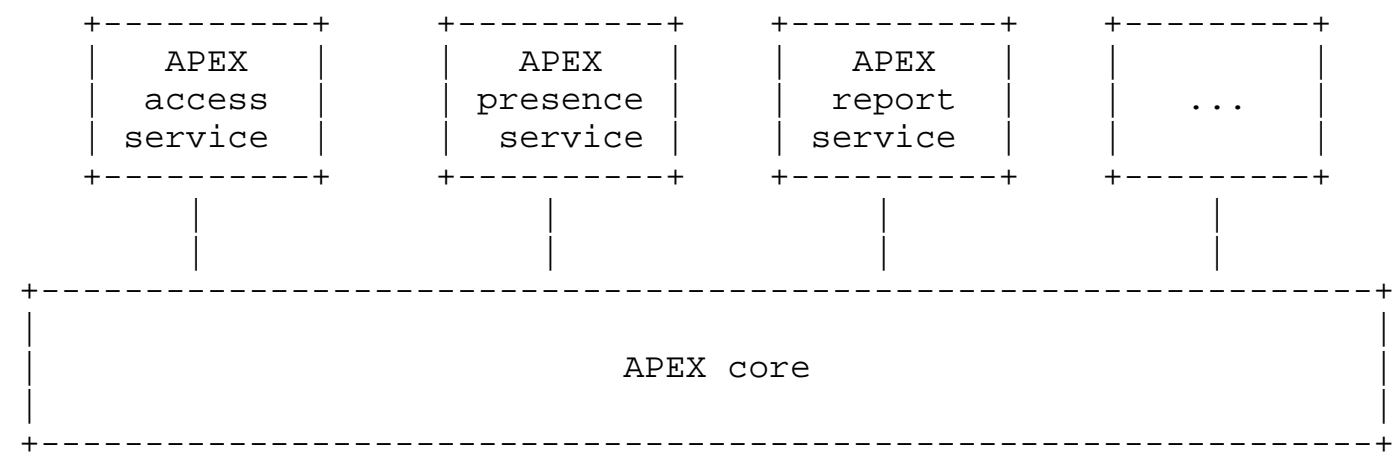

That is, applications communicate with an APEX service by exchanging data with a "well-known endpoint" (WKE).

For example, APEX applications communicate with the report service by exchanging data with the well-known endpoint "apex=report" in the corresponding administrative domain, e.g., "apex=report@example.com" is the endpoint associated with the report service in the "example.com" administrative domain.

The specification of an APEX service must define:

o the WKE of the service;

o the syntax and sequence of messages exchanged with the service;

o what access control tokens are consulted by the service. 
A service registration template (Section 7.2) organizes this information.

Finally, note that within a single administrative domain, the relaying mesh makes use of the APEX access service in order to determine if an originator is allowed to transmit data to a recipient (c.f., Step 5.3 of Section 4.4.4.1).

\subsection{Use of the APEX Core DTD}

The specification of an APEX service may use definitions found in the APEX core DTD (Section 9.1). For example, the reply operation (Section 6.1.2) is defined to provide a common format for responses.

\subsubsection{Transaction-Identifiers}

In using APEX's transaction-identifiers, note the following:

- In the endpoint-relay and relay-relay modes, transactionidentifiers are meaningful only during the lifetime of a BEEP channel.

For example, when an application issues the attach operation, the associated transaction-identifier has meaning only within the context of the BEEP channel used for the attach operation. When the BEEP connection is released, the channel no longer exists and the application is no longer attached to the relaying mesh.

- In contrast, when an application communicates with an APEX service, transaction-identifiers are often embedded in the data that is sent. This means that transaction-identifiers are potentially long-lived.

For example, an application may attach as an endpoint, send data (containing an embedded transaction-identifier) to a service, and, some time later, detach from the relaying mesh. Later on, a second application may attach as the same endpoint, and send data of its own (also containing embedded transaction-identifiers). Subsequently, the second application may receive data from the service responding to the first application's request and containing the transaction-identifier used by the first application.

To minimize the likelihood of ambiguities with long-lived transaction-identifiers, the values of transaction-identifiers generated by applications should appear to be unpredictable. 


\subsubsection{The Reply Element}

Many APEX services make use of a reply operation. Although each service defines the circumstances in which a "reply" element is sent, the syntax of the "reply" element is defined in Section 9.1 .

The "reply" element has a "code" attribute, a "transid" attribute, an optional "xml:lang" attribute, and may contain arbitrary textual content:

o the "code" element specifies a three-digit reply code (c.f., Section 10);

o the "transID" attribute specifies the transaction-identifier corresponding to this reply;

o the "xml:lang" attribute, if present, specifies the language that the element's content is written in; and,

- the textual content is a diagnostic (possibly multiline) which is meaningful to implementers, perhaps administrators, and possibly even users.

6.2 The Report Service

Section 8.5 contains the APEX service registration for the report service:

- Within an administrative domain, the service is addressed using the well-known endpoint of "apex=report".

- Section 9.2 defines the syntax of the operations exchanged with the service.

- A consumer of the service does not initiate communications with the service.

- The service initiates communications by sending data containing the "statusResponse" operation.

If a relay processes a "statusRequest" option (Section 5.1), then it sends data to the originator containing a "statusResponse" element (Section 9.2).

The "statusResponse" element has a "transID" attribute and contains one or more "destination" elements: 
- the "transID" attribute specifies the value contained in the "statusRequest" option; and,

o each "destination" element has an "identity" attribute and contains a "reply" element:

* the "identity" attribute specifies the recipient endpoint that is being reported on; and,

* the "reply" element (Section 6.1.2) specifies the delivery status of that recipient.

7. Registration Templates

7.1 APEX Option Registration Template

When an APEX option is registered, the following information is supplied:

Option Identification: specify the NMTOKEN or the URI that authoritatively identifies this option.

Present in: specify the APEX elements in which the option may appear.

Contains: specify the XML content that is contained within the "option" element.

Processing Rules: specify the processing rules associated with the option.

Contact Information: specify the postal and electronic contact information for the author of the profile.

\subsection{APEX Service Registration Template}

When an APEX service is registered, the following information is supplied:

Well-Known Endpoint: specify the local-part of an endpoint identity, starting with "apex=".

Syntax of Messages Exchanged: specify the elements exchanged with the service.

Sequence of Messages Exchanged: specify the order in which data is exchanged with the service. 
Access Control Tokens: specify the token(s) used to control access to the service (c.f., [10]).

Contact Information: specify the postal and electronic contact information for the author of the profile.

Note that the endpoints "apex=all" and "apex=core" may not be assigned.

7.3 APEX Endpoint Application Registration Template

When an APEX endpoint application is registered, the following information is supplied:

Endpoint Application: specify the subaddress used for an endpoint application, starting with "appl=".

Application Definition: specify the syntax and semantics of the endpoint application identified by this registration.

Contact Information: specify the postal and electronic contact information for the author of the profile.

8. Initial Registrations

8.1 Registration: The APEX Profile

Profile Identification: http://iana.org/beep/APEX

Messages exchanged during Channel Creation: "attach", "bind"

Messages starting one-to-one exchanges: "attach", "bind",

"terminate", or "data"

Messages in positive replies: "ok"

Messages in negative replies: "error"

Messages in one-to-many exchanges: none

Message Syntax: c.f., Section 9.1

Message Semantics: c.f., Section 4.4

Contact Information: c.f., the "Authors' Addresses" section of this memo

Rose, et. al.

Standards Track

[Page 30] 
8.2 Registration: The System (Well-Known) TCP port number for apex-mesh Protocol Number: TCP

Message Formats, Types, Opcodes, and Sequences: c.f., Section 9.1

Functions: c.f., Section 4.4

Use of Broadcast/Multicast: none

Proposed Name: APEX relay-relay service

Short name: apex-mesh

Contact Information: c.f., the "Authors" Addresses" section of this memo

8.3 Registration: The System (Well-Known) TCP port number for apex-edge Protocol Number: TCP

Message Formats, Types, Opcodes, and Sequences: c.f., Section 9.1

Functions: c.f., Section 4.4

Use of Broadcast/Multicast: none

Proposed Name: APEX endpoint-relay service

Short name: apex-edge

Contact Information: c.f., the "Authors' Addresses" section of this memo

8.4 Registration: The statusRequest Option

Option Identification: statusRequest

Present in: APEX's "data" and "recipient" elements

Contains: nothing

Processing Rules: c.f., Section 5.1

Contact Information: c.f., the "Authors' Addresses" section of this memo

Rose, et. al.

Standards Track

[Page 31] 
8.5 Registration: The Report Service

Well-Known Endpoint: apex=report

Syntax of Messages Exchanged: c.f., Section 9.2

Sequence of Messages Exchanged: c.f., Section 6.2

Access Control Tokens: none

Contact Information: c.f., the "Authors' Addresses" section of this memo

9. DTDS

9.1 The APEX Core DTD

$<!--$

DTD for the APEX core, as of 2001-07-09

Refer to this DTD as:

<!ENTITY APEXCORE PUBLIC "-//IETF//DTD APEX CORE//EN" " "> $\circ \mathrm{APEXCORE}$; $-->$

<!ENTITY BEEP PUBLIC "-//IETF//DTD BEEP//EN" " "> $\circ \mathrm{BEEP}$;

$<!--$

DTD data types:

entity syntax/reference example

$=====0===========0==$

APEX endpoint ENDPOINT

entity,

c.f., Section 2.2

fred@example.com

domain, either a FQDN or a literal

DOMAIN C.f., [RFC-2821]

example.com or [10.0.0.1]

seconds

SECONDS $\quad 0.2147483647 \quad 600$

timestamp

TIMESTAMP C.f., 2000-05-15T13:02:00-08:00

unique-identifier

Rose, et. al.

Standards Track

[Page 32] 


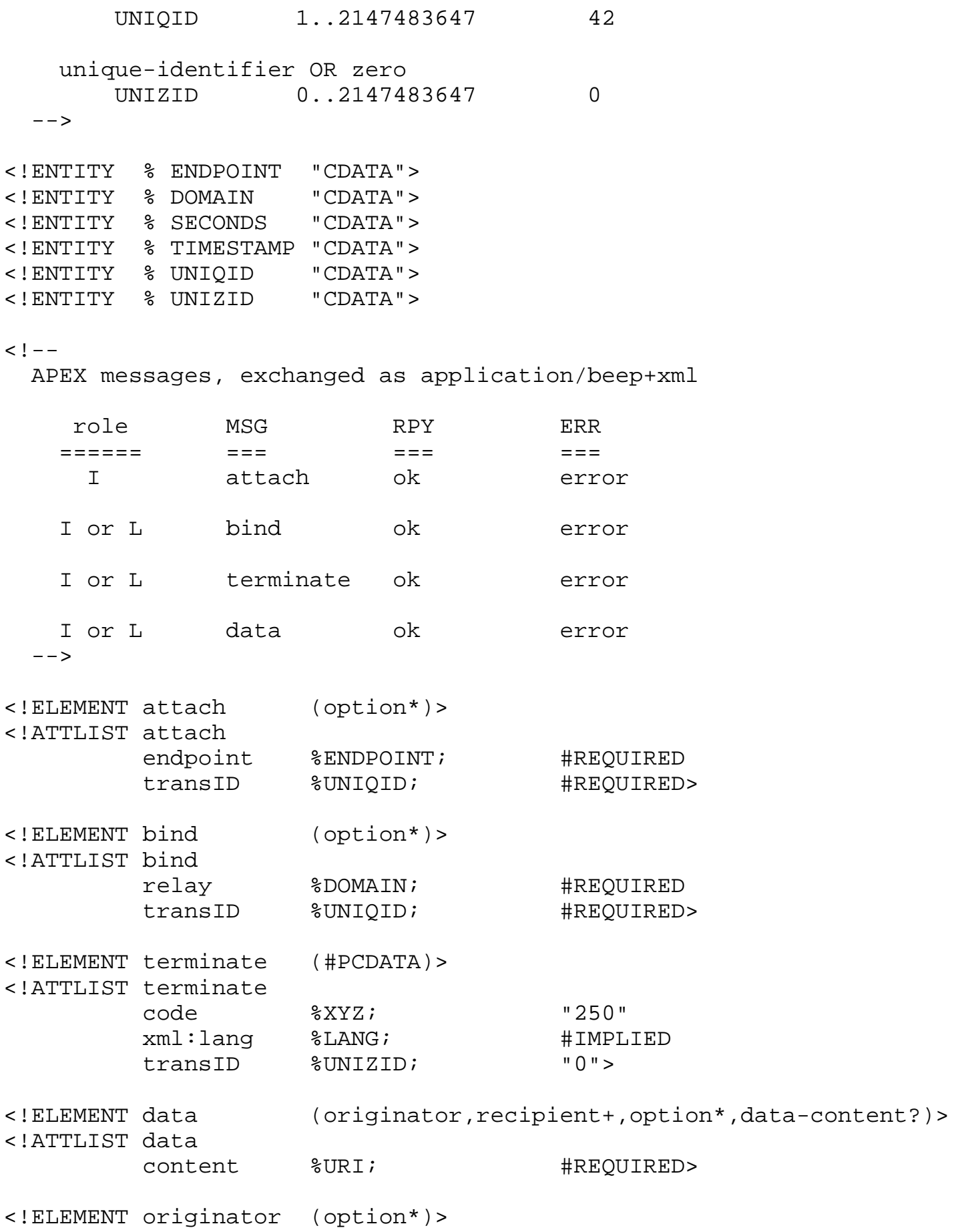




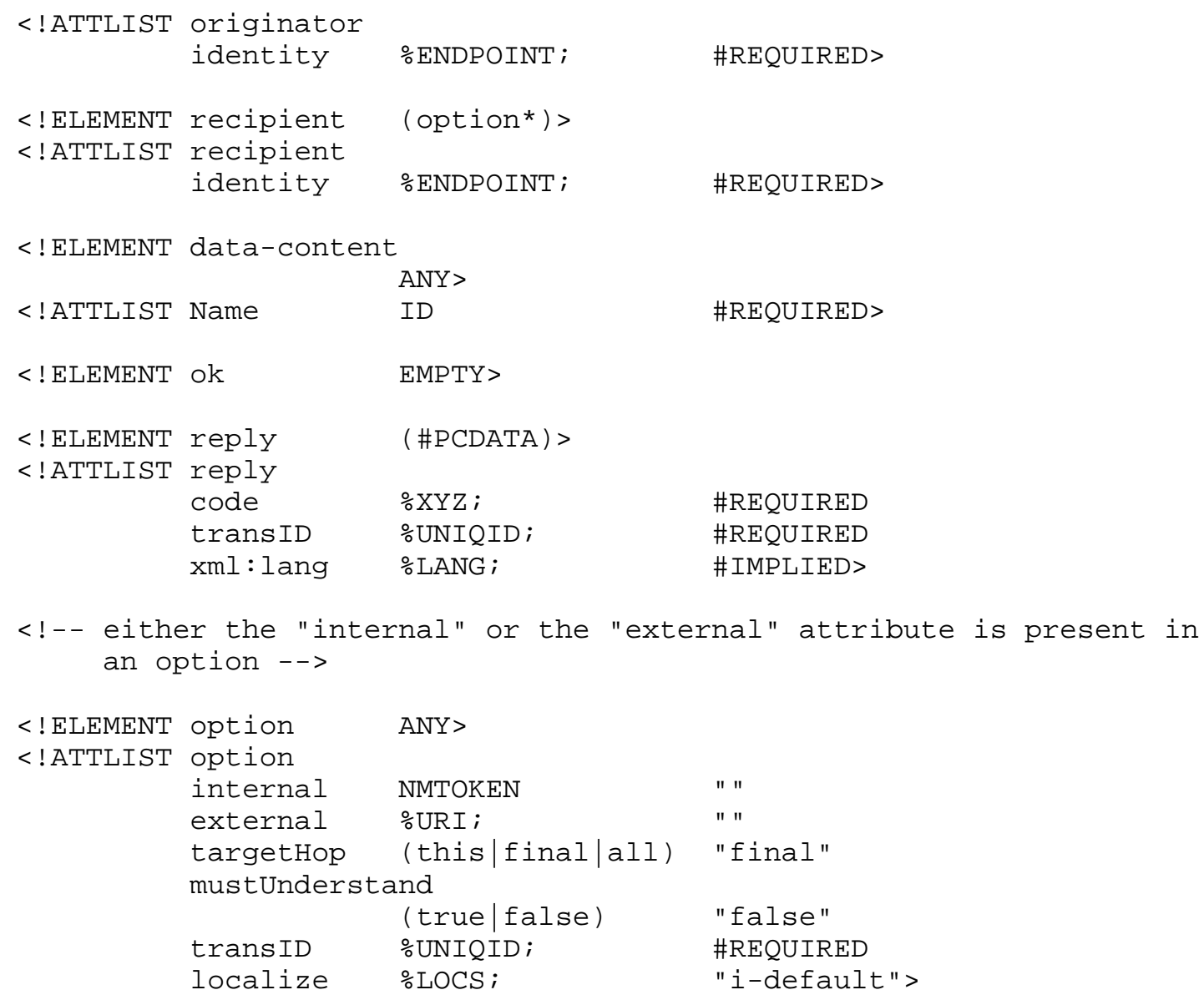




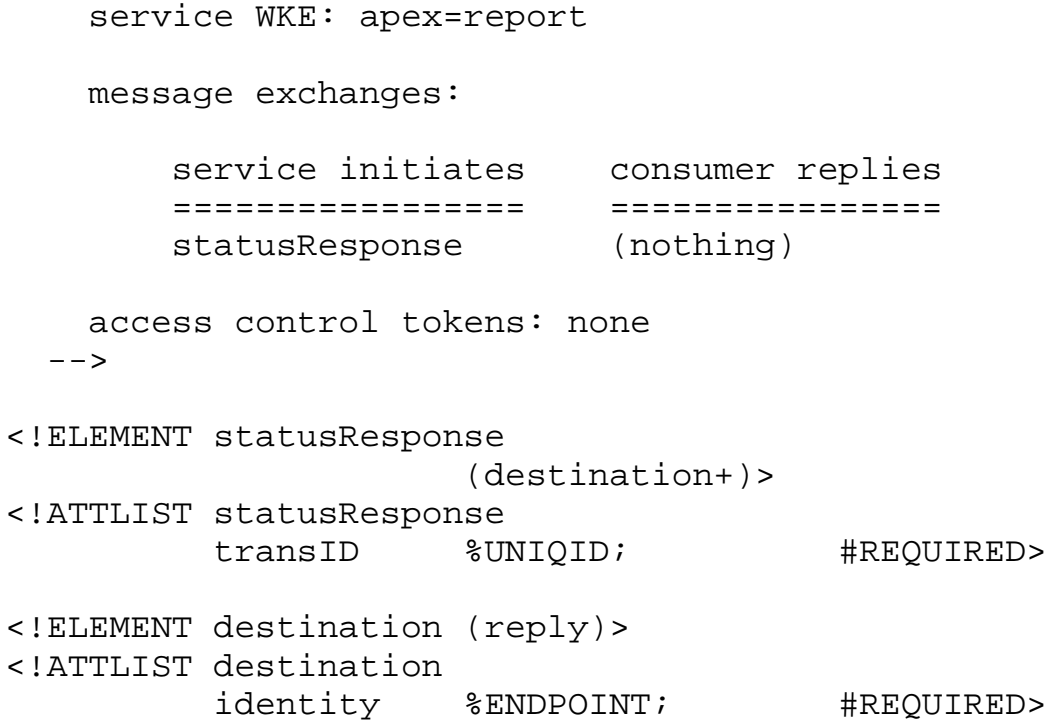

10. Reply Codes

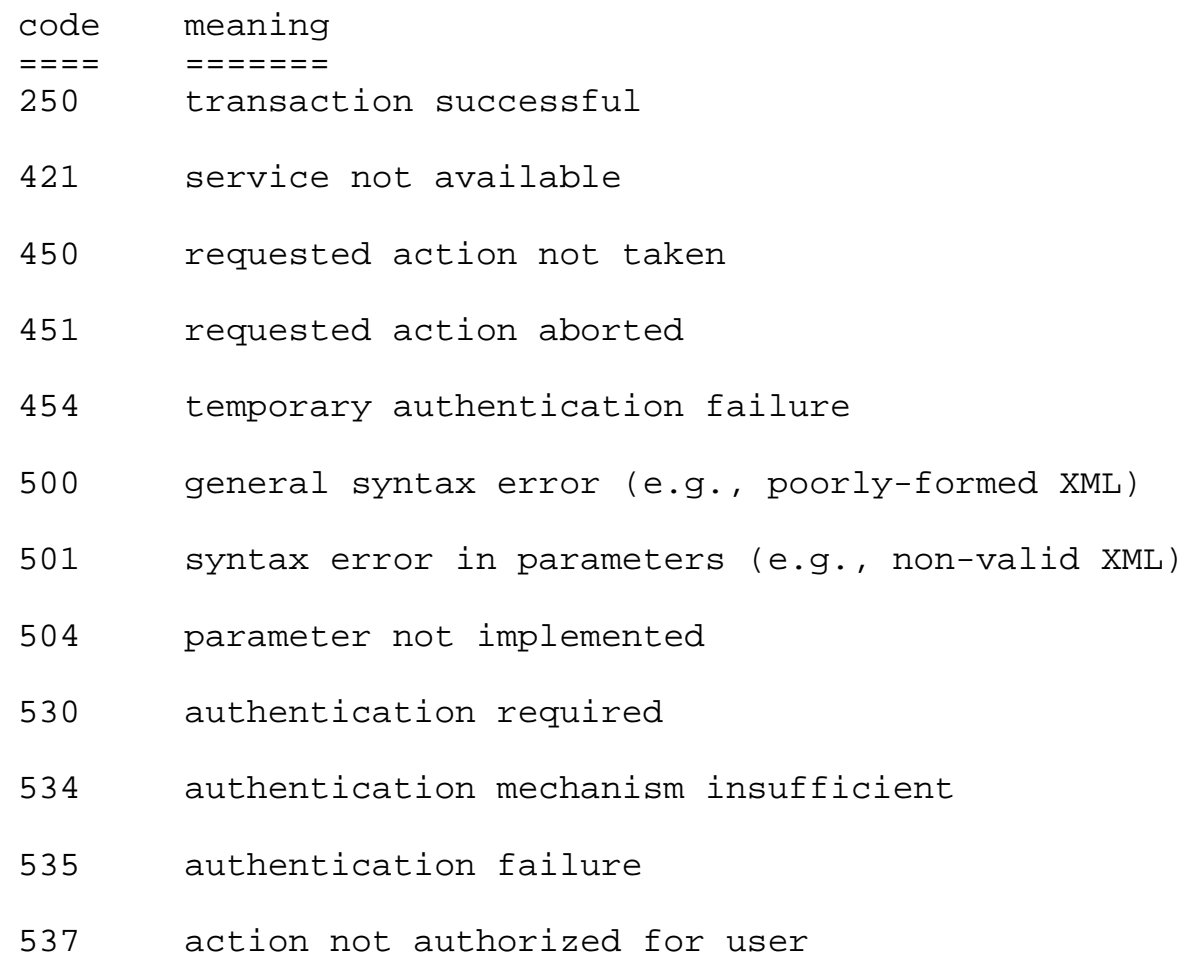




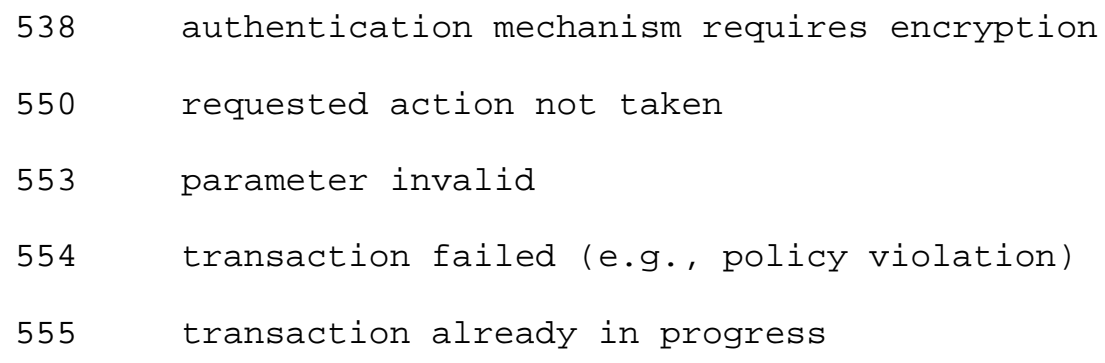

11. Security Considerations

Consult Section 3 and section 4.5 for a discussion of security issues, e.g., relaying integrity.

Although service provisioning is a policy matter, at a minimum, all APEX implementations must provide the following tuning profiles:

for authentication: http://iana.org/beep/SASL/DIGEST-MD5

for confidentiality: http://iana.org/beep/TLS (using the TLS_RSA_WITH_3DES_EDE_CBC_SHA cipher)

for both: http://iana.org/beep/TLS (using the TLS_RSA_WITH_3DES_EDE_CBC_SHA cipher supporting client-side certificates)

Further, APEX endpoint implementations may choose to offer MIME-based security services providing message integrity and confidentiality, such as OpenPGP [13] or S/MIME [14].

Regardless, since APEX is a profile of the BEEP, consult [1]'s Section 9 for a discussion of BEEP-specific security issues.

Finally, the statusRequest option (Section 5.1) may be used to expose private network topology. Accordingly, an administrator may wish to choose to disable this option except at the ingress/egress points for its administrative domain.

References

[1] Rose, M., "The Blocks Extensible Exchange Protocol Core", RFC 3080 , March 2001 .

[2] Crocker, D. and P. Overell, "Augmented BNF for Syntax Specifications: ABNF", RFC 2234, November 1997. 
[3] Klensin, J., "Simple Mail Transfer Protocol", RFC 2821, April 2001 .

[4] Yergeau, F., "UTF-8, a transformation format of Unicode and ISO $10646 "$, RFC 2044, October 1996.

[5] Gulbrandsen, A., Vixie, P. and L. Esibov, "A DNS RR for specifying the location of services (DNS SRV)", RFC 2782, February 2000 .

[6] Berners-Lee, T., Fielding, R. and L. Masinter, "Uniform Resource Identifiers (URI): Generic Syntax", RFC 2396, August 1998 .

[7] Levinson, E., "The MIME Multipart/Related Content-type", RFC 2387, August 1998 .

[8] Levinson, E., "Content-ID and Message-ID Uniform Resource Locators", RFC 2392, August 1998.

[9] Freed, N. and N. Borenstein, "Multipurpose Internet Mail Extensions (MIME) Part One: Format of Internet Message Bodies", RFC 2045, November 1996.

[10] Rose, M., Klyne, G. and D. Crocker, "The Application Exchange (APEX) Access Service", RFC 3341, July 2002.

[11] Rose, M., Klyne, G. and D. Crocker, "The Application Exchange (APEX) Presence Service", Work in Progress.

[12] Newman, C. and G. Klyne, "Date and Time on the Internet: Timestamps", RFC 3339, July 2002 .

[13] Elkins, M., Del Torto, D., Levien, R. and T. Roessler, "MIME Security with OpenPGP", RFC 3156, August 2001.

[14] Ramsdell, B., "S/MIME Version 3 Message Specification", RFC 2633, June 1999. 
Appendix A. Acknowledgements

The authors gratefully acknowledge the contributions of: Jeffrey Altman, Harald Alvestrand, Eric Dixon, Ronan Klyne, Darren New, Chris Newman, Scott Pead, and Bob Wyman.

Appendix B. IANA Considerations

The IANA has registered "APEX" as a standards-track BEEP profile, as specified in section 8.1 .

The IANA has registered "apex-mesh" as a TCP port number, as specified in Section 8.2 .

The IANA has registered "apex-edge" as a TCP port number, as specified in Section 8.3 .

The IANA maintains a list of:

o APEX options, c.f., Section 7.1;

- APEX services, c.f., Section 7.2; and,

- APEX endpoint applications, c.f., Section 7.3.

For each list, the IESG is responsible for assigning a designated expert to review the specification prior to the IANA making the assignment. As a courtesy to developers of non-standards track APEX options and services, the mailing list apexwgeinvisible.net may be used to solicit commentary.

The IANA makes the registrations specified in section 8.4 and section 8.5 . 


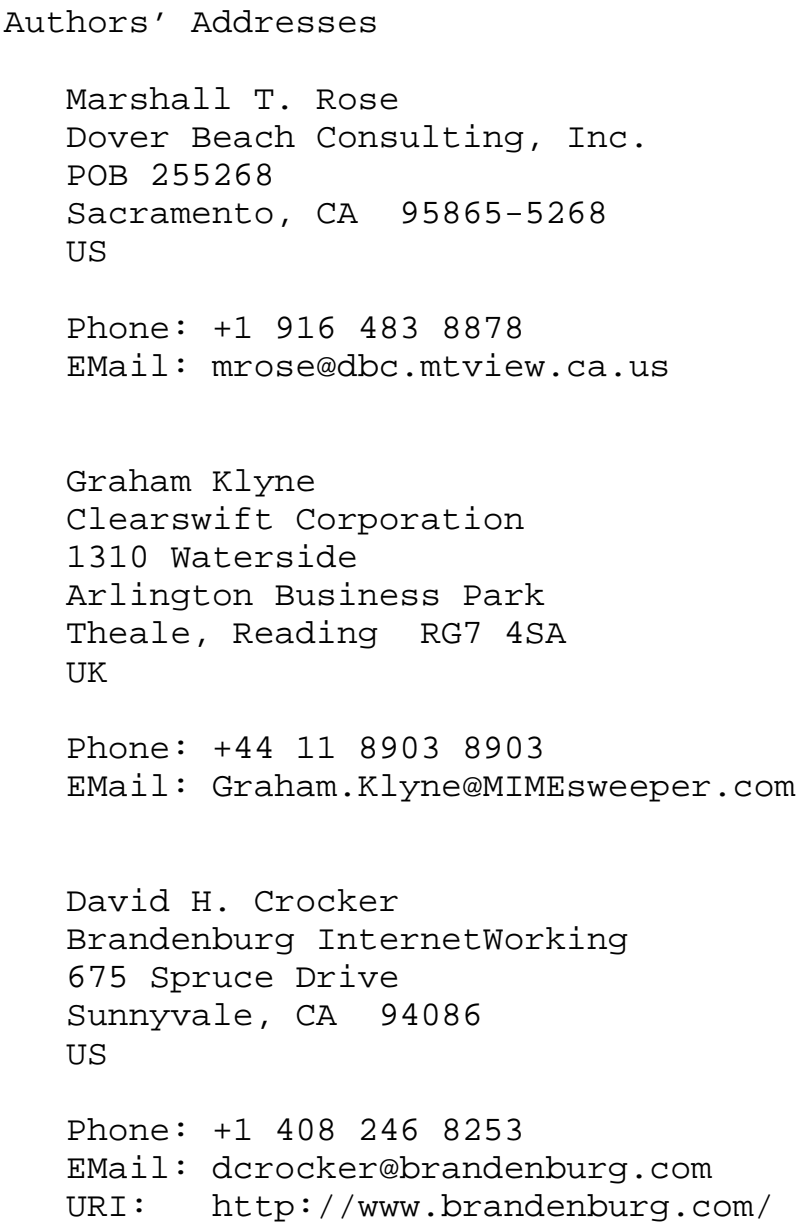


Full Copyright statement

Copyright (C) The Internet Society (2002). All Rights Reserved.

This document and translations of it may be copied and furnished to others, and derivative works that comment on or otherwise explain it or assist in its implementation may be prepared, copied, published and distributed, in whole or in part, without restriction of any kind, provided that the above copyright notice and this paragraph are included on all such copies and derivative works. However, this document itself may not be modified in any way, such as by removing the copyright notice or references to the Internet society or other Internet organizations, except as needed for the purpose of developing Internet standards in which case the procedures for copyrights defined in the Internet Standards process must be followed, or as required to translate it into languages other than English.

The limited permissions granted above are perpetual and will not be revoked by the Internet society or its successors or assigns.

This document and the information contained herein is provided on an "AS IS" basis and THE INTERNET SOCIETY AND THE INTERNET ENGINEERING TASK FORCE DISCLAIMS ALL WARRANTIES, EXPRESS OR IMPLIED, INCLUDING BUT NOT LIMITED TO ANY WARRANTY THAT THE USE OF THE INFORMATION HEREIN WILL NOT INFRINGE ANY RIGHTS OR ANY IMPLIED WARRANTIES OF MERCHANTABILITY OR FITNESS FOR A PARTICULAR PURPOSE.

Acknowledgement

Funding for the RFC Editor function is currently provided by the Internet Society. 